\title{
Colouring crystals with inorganic nanoparticles $\uparrow$
}

Cite this: Chem. Commun., 2014 50,67

Received 14th October 2013,

Accepted 5th November 2013

DOI: $10.1039 / \mathrm{c3cc} 47904 \mathrm{~h}$

www.rsc.org/chemcomm

\author{
Alexander N. Kulak, ${ }^{a}$ Pengcheng Yang, ${ }^{\mathrm{b}}$ Yi-Yeoun Kim, $^{\mathrm{a}}$ Steven P. Armes ${ }^{\mathrm{b}}$ and \\ Fiona C. Meldrum*a
}

A simple, one-pot method is presented whereby gold nanoparticles coated with a zwitterionic diblock copolymer are incorporated within single crystals of calcite. This may provide a versatile alternative to dyeing crystal with organic molecules and could be extended to create a series of new nanocomposite crystals with novel properties.

That single crystals can be coloured through the occlusion of dye molecules within their lattice has been recognised for centuries. ${ }^{1}$ Often giving rise to symmetric patterns of colour, the number of dye and crystal combinations which lead to occlusion within an ionic crystal are typically rather limited and often difficult to predict. Indeed, from 962 experiments in which 26 dyes were crossed with 37 ionic crystals, a determined Retgers identified only four mixed crystals which showed significant levels of incorporation. ${ }^{2}$ The complex process of dye incorporation within crystals is therefore really quite intriguing; in addition to endowing the host crystal with potentially interesting physical and optical properties, it can also provide useful insights into the underlying mechanisms of important phenomena such as crystal growth, habit modification by additives and epitaxy. ${ }^{1}$

The ability to entrap impurities within single crystals extends well beyond dye molecules. Particulate occlusions are often observed within rocks or ice, where these usually form during a freezing transition. ${ }^{3-6}$ Biominerals are also invariably composite materials, whereby organic macromolecules can be embedded within single crystals. ${ }^{7-9}$ In this way, nature engineers materials with poor physical properties such as calcium carbonate to build complex skeletal structures with substantially enhanced fracture toughness. ${ }^{7,10}$ This strategy can also be adopted by the synthetic materials chemist to occlude small molecules ${ }^{11}$ and gel fibres ${ }^{12,13}$ within single crystals of calcite and to encapsulate latex particles within zinc oxide crystals. ${ }^{14,15}$ We have also recently described how remarkably high volume

\footnotetext{
${ }^{a}$ School of Chemistry, University of Leeds, Leeds, LS2 9JT, UK.

E-mail: F.Meldrum@leeds.ac.uk

${ }^{b}$ Dainton Building, Department of Chemistry, University of Sheffield, Brook Hill, Sheffield, Yorkshire, S3 7HF, UK. E-mail: S.P.Armes@sheffield.ac.uk

$\dagger$ Electronic supplementary information (ESI) available: Experimental methods, additional characterisation. See DOI: 10.1039/c3cc47904h
}

fractions of latex particles ${ }^{16}$ and copolymer micelles ${ }^{17}$ can be occluded within calcite single crystals. Here, successful occlusion critically depends on the particle surface chemistry, leading in the latter case to crystals with mechanical properties that rival those of calcite biominerals.

In this paper, we build on these results to develop a novel and potentially quite general strategy for colouring single crystals - based on the incorporation of inorganic nanoparticles, rather than organic molecules (Fig. 1). The occlusion of gold nanoparticles (GNPs) within calcite was identified as a suitable model system, since the gold particles are strongly coloured and they can be readily distinguished from the calcite matrix using electron microscopy. Pure calcite, in turn, is colourless and is known to occlude a wide range of impurities. By tailoring the GNP surface chemistry using an appropriate diblock copolymer, ${ }^{18,19}$ they can be occluded within the host crystal without

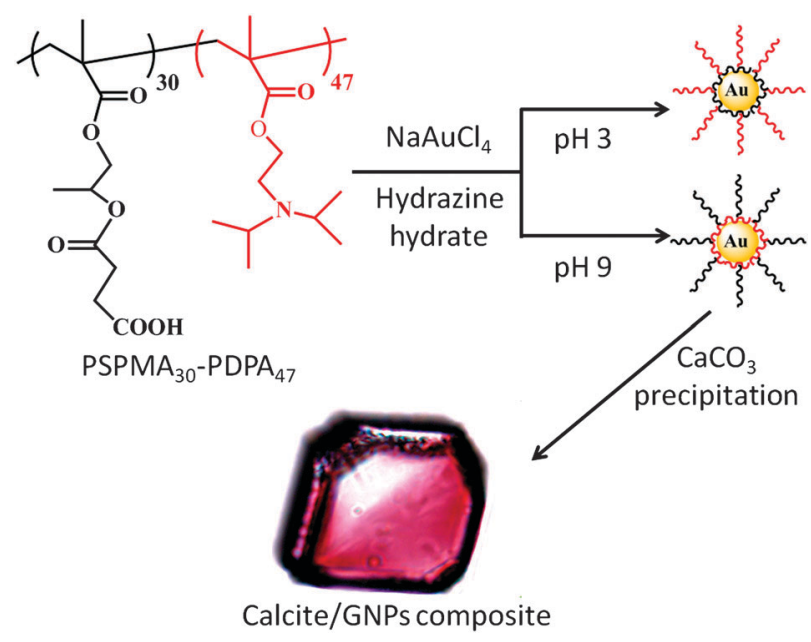

Fig. 1 Schematic diagram showing the structure of the PSPMA ${ }_{30}-\mathrm{PDPA}_{47}$ zwitterionic diblock copolymer used to stabilise the gold nanoparticles (GNPs). The GNPs are synthesised in the presence of this copolymer, whose specific binding mode (whether the PSPMA 30 block or the PDPA 50 block is adsorbed) can be reversed on adjusting the solution $\mathrm{pH}$. Precipitation of $\mathrm{CaCO}_{3}$ in the presence of the GNPs results in their occlusion within single crystals of calcite, producing pink crystals. 
perturbing its single crystal structure, thus changing its intrinsic properties and driving the formation of coloured calcite crystals. Design of a suitable diblock copolymer is therefore fundamental to this approach; one block should adsorb onto the gold nanoparticles, while the other block both promotes binding of the nanoparticle to the crystal and ensures colloidal stability of the nanoparticles in the crystal growth solution via electrosteric stabilisation.

A series of pH-responsive, zwitterionic diblock copolymers, $\mathrm{PSPMA}_{n}-\mathrm{PDPA}_{47}$ (where $n=16,30,50$ and 70), were investigated as coatings for the GNPs. This copolymer structure was selected as the tertiary amine methacrylate-based PDPA block was expected to bind strongly to the GNPs at $\mathrm{pH} 7-9$, which is the $\mathrm{pH}$ window used for $\mathrm{CaCO}_{3}$ formation, while the carboxylate-functionalised PSPMA block was anticipated to provide both effective electrosteric stabilisation of the nanoparticles in the crystal growth solution and promote their incorporation within the calcite crystal. ${ }^{17}$ GNPs were synthesised at $20{ }^{\circ} \mathrm{C}$ from a solution of $\mathrm{PSPMA}_{n}-\mathrm{PDPA}_{47}$ and $\mathrm{NaAuCl}_{4}$, through addition of hydrazine hydrate. During the reduction from $\mathrm{Au}(\mathrm{III})$ to $\mathrm{Au}(0)$, the transparent, yellow solution transformed into a wine-red suspension of copolymer-stabilised GNPs, while an immediate black precipitate of gold was observed in the absence of any copolymer stabiliser. After reduction, the reaction mixture was stirred overnight, before being dialysed against copious quantities of deionised water.

The colloidal stability of the GNPs was strongly dependent on the precise copolymer structure. Thus those sols prepared using $\mathrm{PSPMA}_{n}-\mathrm{PDPA}_{47}(n=50,70)$ were not stable during dialysis, and sedimented as a grey-purple powder. In contrast, GNPs prepared using $\mathrm{PSPMA}_{n}-\mathrm{PDPA}_{47}(n=16$ or 30 ) exhibited remarkable stability, not only during dialysis but also at $\mathrm{pH}$ values as low as 1.5 and as high as 12 ( $\mathrm{pH}$ adjusted using $\mathrm{HCl}$ and $\mathrm{NaOH}$, respectively). No precipitation was observed for at least two weeks over this wide $\mathrm{pH}$ range. Characterisation of these particles using transmission electron microscopy (TEM) indicated that the gold particles themselves had diameters of $\sim 10-70 \mathrm{~nm}$, with a mean value of 10-20 nm (Fig. S1, ESI $\dagger$ ). Measurement of the sizes of the $\mathrm{PSPMA}_{30}-\mathrm{PDPA}_{47}$-stabilised nanoparticles in solution using dynamic light scattering (DLS) indicated somewhat larger diameters due to the steric stabiliser

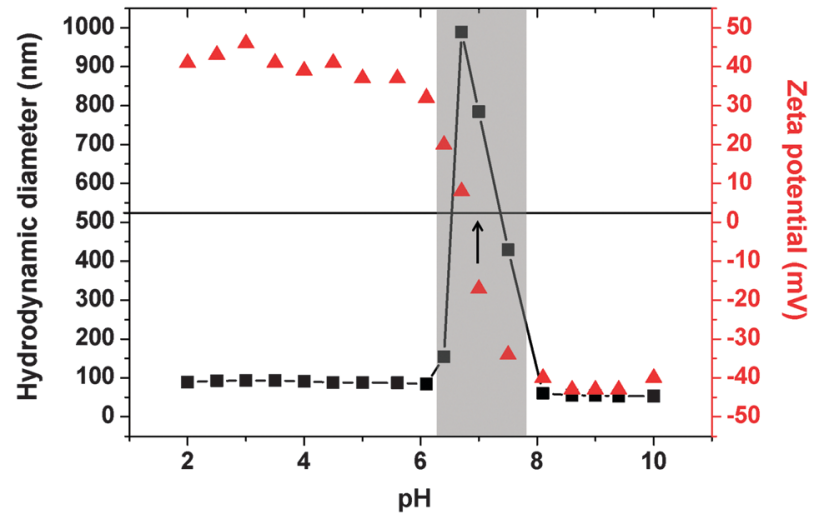

Fig. 2 Mean hydrodynamic diameter and zeta potential vs. $\mathrm{pH}$ curves obtained for a $0.1 \mathrm{w} / \mathrm{v} \%$ aqueous dispersion of gold nanoparticles stabilised using a PSPMA $30-P_{3 P A}$ zwitterionic diblock copolymer. The isoelectric point is at approximately $\mathrm{pH} 6.8$ (see arrow) and the grey shaded area indicates the region of colloidal instability. thickness layer (see Fig. 2). Zeta potential measurements indicated that they possessed cationic surface character at low $\mathrm{pH}(+46 \mathrm{mV}$ at $\mathrm{pH}$ 3.0) and weakly anionic surface character at high $\mathrm{pH}(-43 \mathrm{mV}$ at $\mathrm{pH}$ 9.0). Thus the GNPs prepared using these PSPMA-PDPA diblock copolymers exhibit similar properties to the 'schizophrenic' copolymer micelles alone (Fig. S2, ESI $\dagger$ ).

The use of these copolymer-stabilised GNPs to colour calcite crystals was then investigated. GNPs stabilised with either PSPMA ${ }_{16}$ $\mathrm{PDPA}_{47}$ or $\mathrm{PSPMA}_{30}-\mathrm{PDPA}_{47}$ gave very similar results, and thus only data using $\mathrm{PSPMA}_{30}-\mathrm{PDPA}_{47}$ copolymer is presented here for simplicity. $\mathrm{CaCO}_{3}$ was precipitated from aqueous $\mathrm{CaCl}_{2}$ solution $\left(\left[\mathrm{Ca}^{2+}\right]=\right.$ $3.0 \mathrm{mM}$ ) in the presence of $0.30 \mathrm{~g} \mathrm{dm}^{-3} \mathrm{PSPMA}_{30}-\mathrm{PDPA}_{47}$-stabilised GNPs using the ammonium carbonate diffusion method. ${ }^{20}$ Crystals were isolated from solution after one day and were rinsed with dilute aqueous $\mathrm{HCl}$ to remove any surface-adsorbed GNPs. In contrast to the colourless calcite crystals precipitated in control experiments (Fig. 3a and Fig. S3a, ESI $\dagger$ ), the $\mathrm{GNP} / \mathrm{CaCO}_{3}$ crystals were visibly pink in colour (Fig. 3b and Fig. S3b, ESI $\dagger$ ). Further examination with scanning electron microscopy (SEM) showed that these coloured crystals were primarily rhombohedral calcite with roughened surfaces (Fig. 3c), where the polymorph was confirmed using Raman microscopy and powder XRD (Fig. S4, ESI $\dagger$ ). Unfortunately, the gold content within the host crystal was too low for identification using powder XRD. In contrast, gold nanoparticles synthesised in the absence of any copolymer using the standard citrate reduction rapidly aggregated when added to $\mathrm{CaCl}_{2}$ solution.

The quantity and location of GNPs occluded within the calcite crystals was also investigated. Atomic absorption spectroscopy, which was performed after dissolving the hybrid crystals in aqua regia, showed that the $\mathrm{CaCO}_{3}$ crystals occluded $\sim 3 \mathrm{wt} \%$ of gold particles. The fracture surfaces of the crystals were also characterised using SEM after grinding crystals. Compared to pure calcite crystals, which fracture to give smooth surfaces, the cross-sections of the calcite-gold hybrid crystals were rough, providing strong evidence for particle incorporation (Fig. S5, ESI $\dagger$ ). This was supported by EnergyDispersive X-Ray (EDX) analysis, which revealed the presence of gold (Fig. 3d). Finally, the location of the GNPs within the calcite lattice was determined via TEM by examining thin sections, which were prepared using Focussed Ion Beam (FIB) (Fig. 3e). No evidence for aggregation of the GNPs within the crystal was obtained, as was confirmed by the calcite composite crystals being pink rather than blue in colour. These studies also confirmed that the GNPs did not disrupt the crystal lattice, as evidenced by selected area electron diffraction which yielded single crystal type patterns (Fig. 3f). High resolution TEM images also showed continuous lattice fringes (Fig. $3 g$ and Fig. S6, ESI $\dagger$ ) while EDX analysis of this sample area confirmed that the occluded particles were indeed gold (Fig. 3h).

The mechanisms by which impurities are incorporated within a crystal vary according to their physical size and chemical structure. The occlusion of (micron- to millimetre-sized) particles during a freezing transition has been widely studied both experimentally and theoretically and depends on many factors, including the thermal diffusivity of the particle and melt, wetting of the crystal face by the particles, the viscosity of the melt, the rate of crystallisation and the particle size. ${ }^{3-6}$ At much shorter length scales, small molecules are believed to bind stereospecifically to step edges or selectively to 

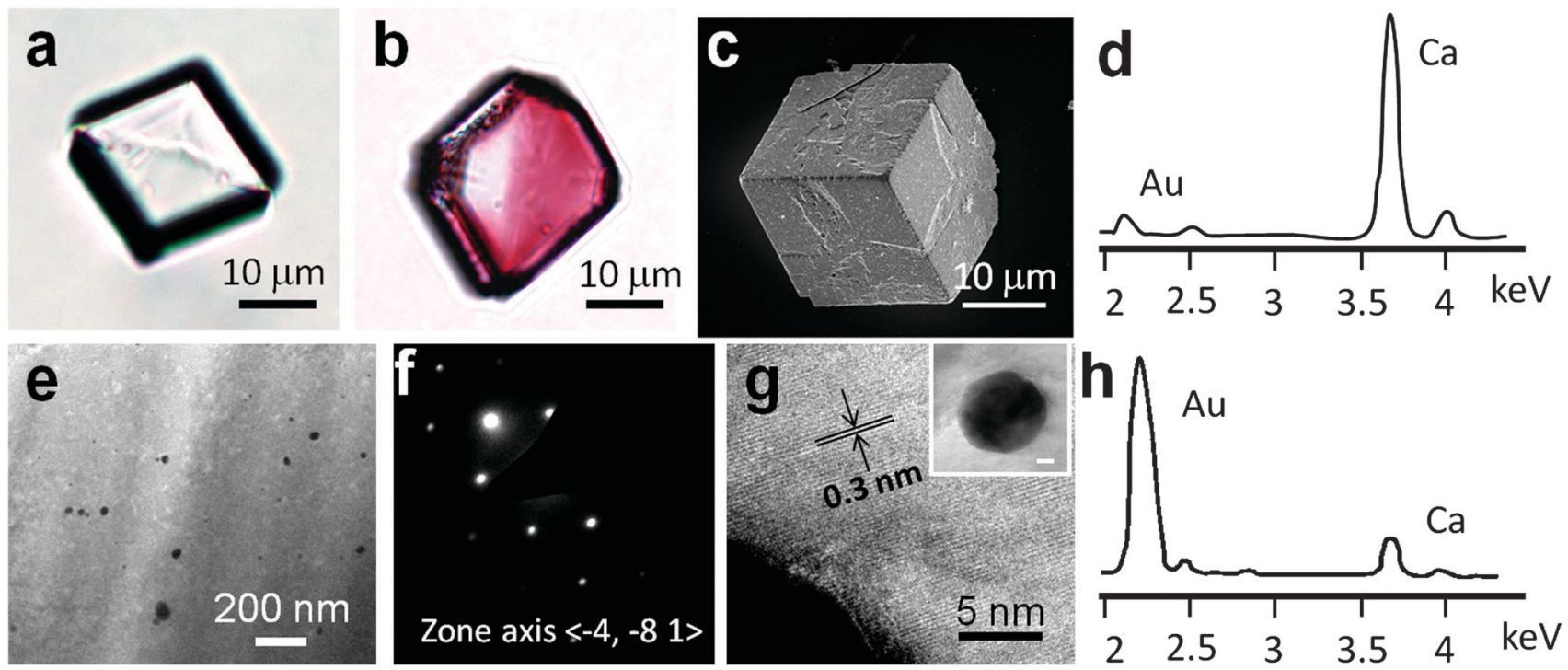

Fig. 3 (a) Optical micrograph of a colourless calcite crystal precipitated in the absence of GNPs. (b-h) Analysis of calcite-GNP hybrid crystals. (b) An optical micrograph of a pink calcite crystal, indicating occlusion of the GNPs, (c) an SEM image showing rough surfaces, (d) an EDX spectrum of a cross-section through a fractured crystal, (e) TEM image of a thin section, cut using FIB, showing GNPs embedded within the crystal, (f) the corresponding selected area electron diffraction pattern, which confirms that the calcite remains a single crystal, (g) a high resolution TEM image of a GNP embedded in the crystal, showing lattice fringes from $\{104\}$ planes and (h) the corresponding EDX spectrum of this area.

specific crystal faces, where they can be subsequently overgrown. ${ }^{21}$ This mechanism also appears to operate during the occlusion of proteins in biominerals, where synchrotron XRD experiments have revealed a remarkable correlation between defect patterns caused by protein occlusion and the gross morphologies of single crystals. ${ }^{22,23}$ The copolymer-stabilised gold nanoparticles used here are substantially larger than proteins and their occlusion is determined by the strength of adsorption to the active kink and step sites, where they compete with incoming crystal growth units. ${ }^{6,24}$ This therefore suggests the ability to tailor the degree of nanoparticle occlusion through appropriate design of the adsorbed copolymer; this hypothesis will be tested in future work.

In conclusion, nanoparticle occlusion can provide an effective route to colouring single crystals. While many crystals (including calcite) change colour when doped with specific impurity ions, a full spectrum of colours is seldom achievable using this approach. Similarly, while some crystals can occlude a wide range of organic dye molecules, others (including calcite) have proven extremely resistant to dyeing. ${ }^{1}$ Our new strategy, where crystals are precipitated in the presence of copolymer-stabilised nanoparticles as simple growth additives, is expected to be extremely versatile in that a single copolymer could be used to drive the occlusion of a range of nanoparticles - selected for their colours based on size and composition - in the desired host crystal. Finally, it is envisaged that the ability to incorporate inorganic nanoparticles within single crystals will facilitate identification of the fundamental design rules that dictate the incorporation of impurity species within crystals, and provide a novel route to the generation of composite crystals with novel properties.

We thank the EPSRC for financial support via grants EP/G00868X/1 and EP/K006304/1 (AK, FCM and SPA). This work was also supported by an EPSRC Leadership Fellowship
(EP/H005374/1; FCM, YYK) and an ERC Advanced Investigator grant (PISA 320372; SPA).

\section{Notes and references}

1 B. Kahr and R. W. Gurney, Chem. Rev., 2001, 101, 89.

2 J. W. Retgers, Z. Phys. Chem., 1893, 12, 583.

3 R. Asthana and S. N. Tewari, J. Mater. Sci., 1993, 28, 5414.

4 A. W. Rempel and M. G. Worster, J. Cryst. Growth, 1999, 205, 427.

5 D. Coupard, F. Girot and J. M. Quenisset, J. Mater. Sci., 1996, 31, 5305.

6 A. A. Chernov, Springer Series in Solid State Sciences, Springer-Verlag, 1984.

7 H.-Y. Li, H. L. Xin, M. E. Kunitake, E. C. Keene, D. A. Muller and L. A. Estroff, Adv. Funct. Mater., 2011, 21, 2028.

8 J. W. C. Dunlop and P. Fratzl, inAnn. Rev. Mater. Res., ed. D. R. Clarke, M. Ruhle and F. Zok, 2010, pp. 1-24.

9 F. C. Meldrum and H. Colfen, Chem. Rev., 2008, 108, 4332.

10 S. Weiner, L. Addadi and H. D. Wagner, Mater. Sci. Eng., C, 2000, 11, 1. 11 S. Borukhin, L. Bloch, T. Radlauer, A. R. Hill, A. H. Fitch and B. Pokroy, Adv. Funct. Mater., 2012, 22, 2416.

12 H.-Y. Li, H. L. Xin, D. A. Muller and L. A. Estroff, Science, 2009, 326, 1244.

13 H.-Y. Li and L. A. Estroff, Adv. Mater., 2009, 21, 470.

14 R. Munoz-Espi, Y. Qi, I. Lieberwirth, C. M. Gomez and G. Wegner, Chem.-Eur. J., 2006, 12, 118.

15 R. Munoz-Espi, A. Chandra and G. Wegner, Cryst. Growth Des., 2006, 1584.

16 Y. Y. Kim, L. Ribeiro, F. Maillot, O. Ward, S. J. Eichhorn and F. C. Meldrum, Adv. Mater., 2010, 22, 2082.

17 Y. Y. Kim, K. Ganesan, P. Yang, A. N. Kulak, S. Borukhin, S. Pechook, L. Ribeiro, R. Kröger, S. J. Eichhorn, S. P. Armes, B. Pokroy and F. C. Meldrum, Nat. Mater., 2011, 10, 890.

18 J. Shan and H. Tenhu, Chem. Commun., 2007, 4580.

19 S. Y. Liu, J. V. M. Weaver, M. Save and S. P. Armes, Langmuir, 2002, 18,8350 .

20 J. Ihli, P. Bots, A. Kulak, L. G. Benning and F. C. Meldrum, Adv. Funct. Mater., 2013, 23, 1965.

21 C. A. Orme, A. Noy, A. Wierzbicki, M. T. McBride, M. Grantham, H. H. Teng, P. M. Dove and J. J. DeYoreo, Nature, 2001, 411, 775.

22 A. Berman, J. Hanson, L. Leiserowitz, T. F. Koetzle, S. Weiner and L. Addadi, Science, 1993, 259, 776.

23 J. Aizenberg, J. Hanson, M. Ilan, L. Leiserowitz, T. F. Koetzle, L. Addadi and S. Weiner, FASEB J., 1995, 9, 262.

24 L. Stappers and J. Fransaer, J. Electrochem. Soc., 2006, 153, C472. 